\title{
Une goutte, deux vitesses La dynamique surprenante d'une goutte d'eau sur un élastomère silicone
}

Aurélie Hourlier-Fargette (aurelie.fargette@dalembert.upmc.fr) et Sébastien Neukirch Institut Jean Le Rond d'Alembert, UMR CNRS 7190, Université Pierre et Marie Curie, 4 place Jussieu, 75252 Paris Cedex 05

Une goutte d'eau dévale généralement un plan incliné à vitesse constante.

\section{Néanmoins, nous avons}

observé que deux allures successives peuvent exister lorsque la goutte dévale sur certains élastomères silicones. Ces matériaux, très utilisés dans l'industrie et la recherche, sont un outil essentiel en microfluidique par exemple. La plupart des élastomères silicones commerciaux sont constitués d'un réseau de chaînes de polymères réticulées, c'est-à-dire interconnectées, mais contiennent également une petite proportion de chaînes libres, non réticulées.

\section{Notre enquête pour comprendre}

l'origine de la dynamique

étonnante d'une goutte sur un tel matériau va nous mener sur leurs traces.

Les termes suivis d'un astérisque sont explicités dans le glossaire, p. 17.

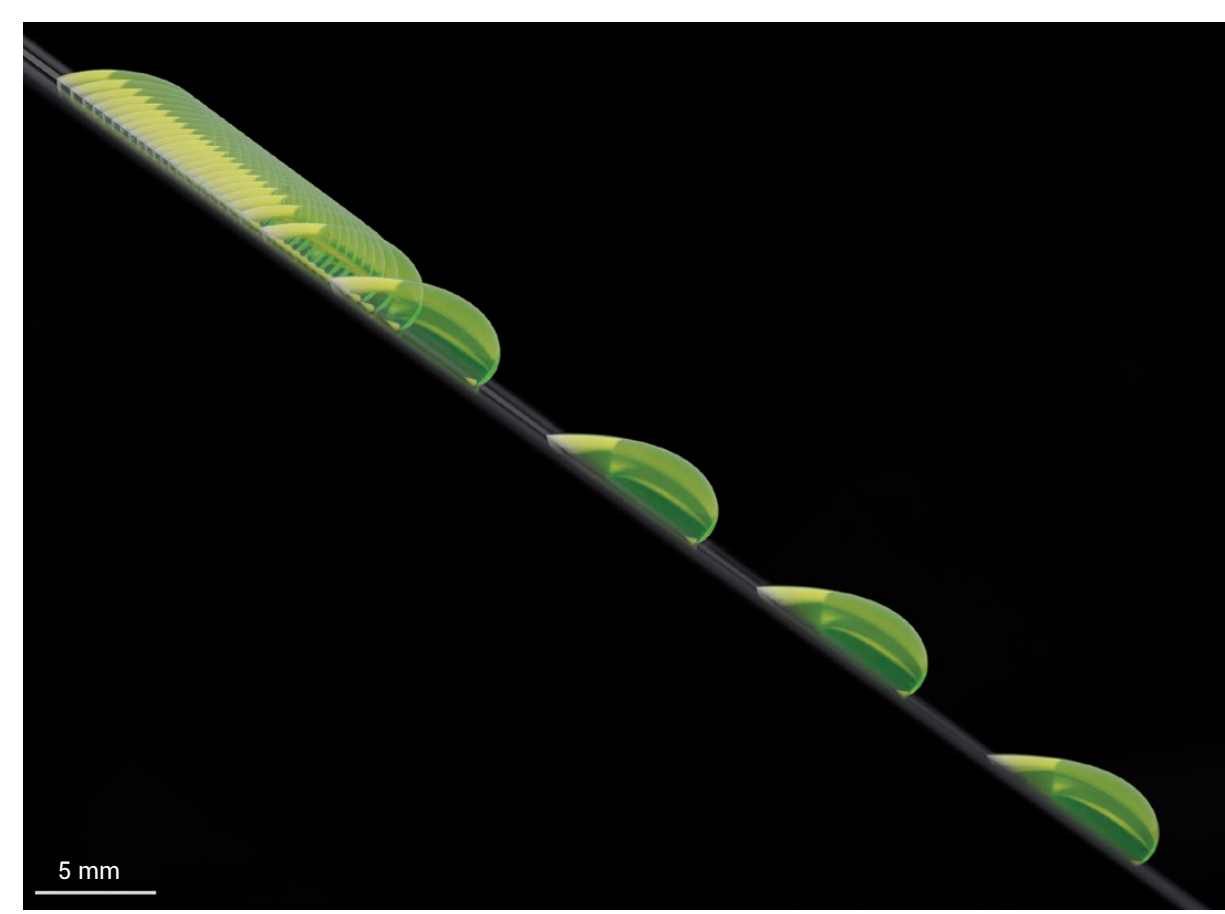

1. Chronophotographie d'une goutte d'un mélange eau-glycérol (colorée pour une meilleure visualisation) dévalant sur un plan incliné en élastomère silicone (polydiméthylsiloxane, PDMS). La taille de la goutte est de quelques millimètres et l'intervalle de temps entre deux images est égal à une seconde. On observe clairement deux régimes distincts, caractérisés par deux vitesses constantes, atteints successivement par la goutte. La deuxième vitesse est plus grande que la première.

À l'interface entre ingénierie, physique, chimie et biologie, les matériaux mous, et en particulier les élastomères ${ }^{\star}$ silicones, sont couramment employés pour fabriquer des microsystèmes à l'aide de techniques de prototypage rapide. Le polydiméthylsiloxane (PDMS) est en particulier très prisé en microfluidique pour la fabrication de microcanaux, dans lesquels des expériences de mécanique des fluides, de chimie ou de biologie sont réalisées. Ce matériau possède de nombreuses qualités : il est transparent, facile à mouler, perméable aux gaz, flexible et peu onéreux. En revanche, certains de ses défauts ont déjà été mentionnés dans la littérature scientifique, comme l'absorption de solvants dans le matériau ou la fuite de molécules issues du PDMS dans le liquide s'écoulant dans les microcanaux [1], affectant les liquides ou les cellules présents dans ces canaux.

C'est à l'interaction entre ces élastomères silicones et les liquides que nous nous intéressons ici. Pour ce faire, nous observons le comportement d'une goutte d'eau sur un plan incliné en PDMS. 


\section{\> \\ Une dynamique de goutte surprenante}

La dynamique d'une goutte sur un plan incliné rigide (en verre traité, par exemple) a fait l'objet de nombreuses études dans la littérature, en particulier depuis les années 1950, mais reste un sujet d'actualité [2]. Posons une goutte sur une plaque rigide horizontale. Si nous penchons la plaque au-delà d'un angle critique, la goutte dévale. Après un bref régime transitoire, elle atteint une vitesse constante déterminée par la compétition entre le poids de la goutte, les forces de tension de surface ${ }^{\star}$ et la dissipation visqueuse dans la goutte.

Remplaçons maintenant la plaque rigide par une plaque en PDMS et étudions le dévalement de gouttes d'eau (ou de mélange eau-glycérol) sur cette plaque inclinée. Nous observons alors une dynamique de dévalement étonnante où sont présents deux régimes successifs, caractérisés par deux vitesses constantes distinctes (figures 1 et 2) [3]. Ce résultat est inattendu et laisse à penser que les interactions entre élastomères silicones et liquides aqueux sont encore mal comprises.

\section{Enquête à l'échelle macroscopique...}

Une première observation consiste à suivre le mouvement du liquide dans la goutte, de façon à savoir si celle-ci roule ou glisse sur le plan incliné, dans chacun des deux régimes de vitesse. En utilisant des particules de cacao comme traceurs dans la goutte, nous observons dans les deux régimes que le mouvement à l'intérieur de la goutte est un mouvement de rotation, dit « en chenillette" (fig. 3). Le changement de vitesse ne s'explique donc pas par un changement du type d'écoulement dans la goutte.

Une deuxième manipulation consiste à renverser l'expérience lorsqu'une goutte est arrivée en bas de la plaque d'élastomère, pour observer la dynamique plusieurs fois avec la même plaque et la même goutte. La première descente d'une goutte est complètement différente des suivantes ! Durant la première descente, nous observons deux régimes de vitesse, tandis que durant les descentes suivantes, la goutte dévale avec une vitesse unique, égale à celle du second régime de la première descente.

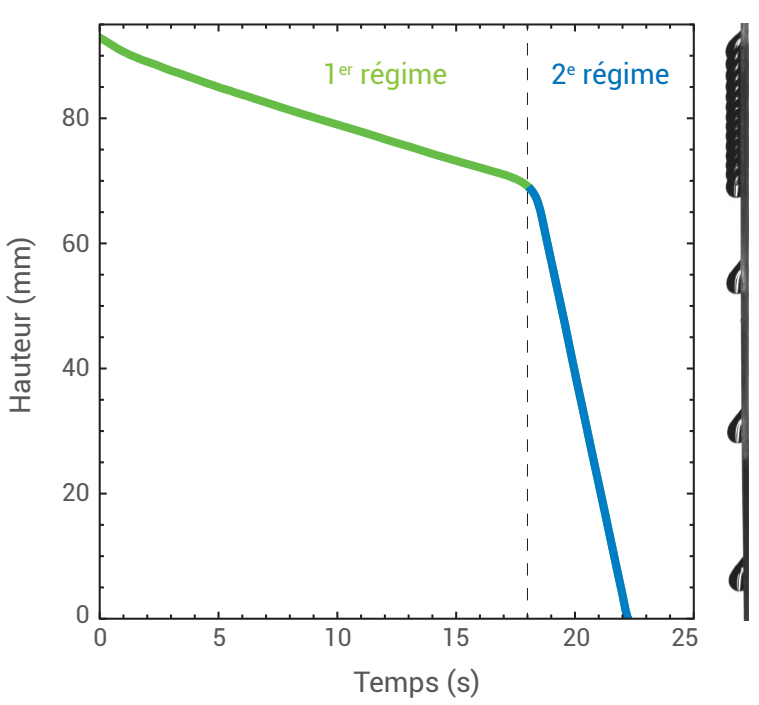

2. Altitude en fonction du temps pour une goutte d'un mélange eau-glycérol déposée puis dévalant sur une plaque verticale en PDMS (schématisée à droite de la figure).
La composition de la goutte est donc probablement modifiée lors de la première descente : ceci nous amène à nous pencher sur la structure de l'élastomère silicone. Le comportement observé à l'échelle macroscopique pourrait tirer son origine de détails microscopiques, à l'échelle du réseau de polymères constituant l'élastomère silicone.

\section{.... et à l'échelle microscopique}

Un élastomère silicone comme le PDMS est composé de chaînes de polymères attachées par des nœuds, appelés points de réticulation, ainsi que d'un petit nombre de chaînes libres, non réticulées. Pour tester l'influence de la présence de ces chaînes

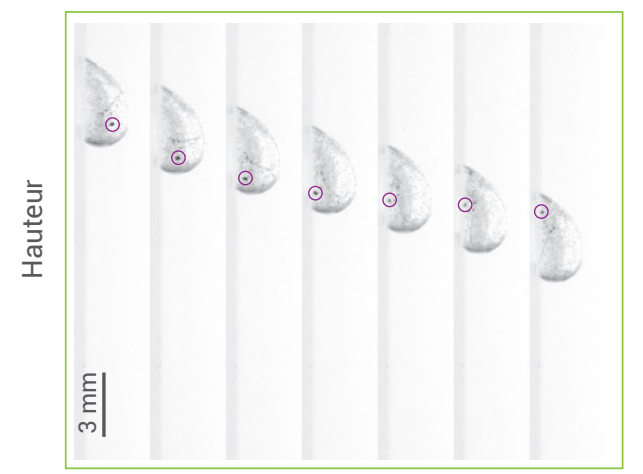

Temps libres de silicone, nous avons comparé la dynamique d'une goutte sur un élastomère silicone contenant des chaînes libres et sur un élastomère n'en contenant pas. En effet, le PDMS peut être débarrassé de ses chaînes libres en utilisant une procédure de lavage grâce à un solvant gonflant fortement la matrice, du toluène par exemple [4]. Lorsque l'élastomère silicone est débarrassé de ses chaînes libres, la goutte dévale avec une vitesse unique, rapidement atteinte après déposition. La présence de chaînes non réticulées est donc décisive dans la dynamique surprenante observée sur un élastomère non lavé. On peut également reproduire le phénomène de double régime en ajoutant des chaînes libres (de l'huile silicone commerciale) à un élastomère lavé.

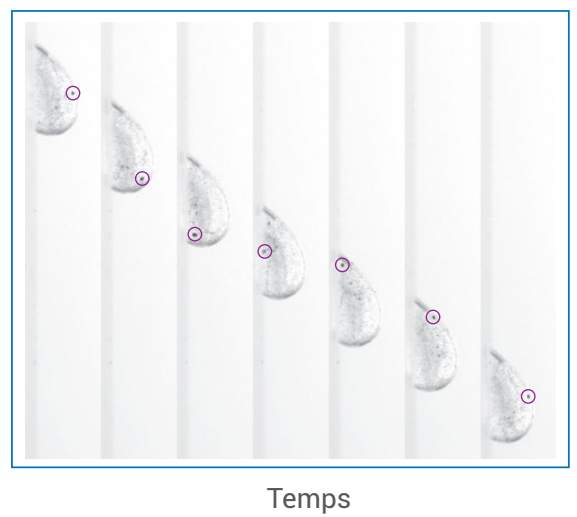

3. Observation de l'écoulement dans la goutte lors du premier régime (à gauche) et du second régime (à droite). Des particules de cacao (entourées par un cercle violet sur la figure) permettent de visualiser un mouvement de rotation dans la goutte pour chacun des deux régimes. Une transition glissement/roulement pour expliquer l'existence des deux régimes de vitesse est donc exclue. La taille de la goutte est de quelques millimètres, et l'intervalle de temps entre deux images est égal à une seconde pour la série correspondant au premier régime (à gauche) et à 0,2 seconde pour la série correspondant au deuxième régime (à droite). 


\section{Des chaînes non réticulées collectées par la goutte}

Comment ces chaînes peuvent-elles interagir avec la goutte ? Au niveau de la ligne de contact entre l'eau, l'air et le PDMS (appelée ligne triple), la force de tension de surface à l'interface eau-air qui s'exerce sur le PDMS peut contribuer à l'extraction des chaînes se plaçant ensuite à l'interface eau-air sur la goutte. Une étude a déjà montré que des chaînes libres pouvaient être extraites d'un gel silicone par adhésion lorsqu'une microbille de verre est posée sur le gel [5]. Dans notre expérience, la bille est remplacée par une goutte, et les forces d'adhésion par la capillarité.

Les propriétés de mouillage de l'huile silicone sur l'eau sont telles que les molécules de silicone sont susceptibles de se placer à l'interface eau-air sur la goutte. S'il y a extraction de chaînes non réticulées, une goutte dévalant sur un élastomère silicone devrait alors collecter une petite fraction de chaînes libres lors de sa descente. Une si petite quantité d'huile silicone est invisible à l'œil nu sur une goutte unique. Cependant, en collectant 1500 gouttes dans un bécher après leur descente sur un plan incliné en PDMS, nous observons des taches d'huile à la surface de l'eau, ce qui confirme notre hypothèse. Aucune trace d'huile n'est visible dans l'expérience témoin réalisée de la même manière sur un plan incliné en PDMS lavé. Chaque goutte collecte donc un peu d'huile silicone lors de sa descente sur un élastomère contenant des chaînes libres.

\section{Discussion : pour aller plus loin}

Si les chaînes libres sont collectées progressivement par la goutte, pourquoi la transition de vitesse est-elle si brutale ? Un paramètre intéressant que nous pouvons mesurer est la tension de surface à l'interface eau-air, en déterminant la force nécessaire pour arracher de la surface de l'eau un cylindre partiellement immergé. Ces mesures nous montrent que (i) dans le premier régime d'une goutte d'eau dévalant sur du PDMS, la valeur de la tension de surface de la goutte est celle de l'eau pure, (ii) que dans le second régime la tension de surface de la goutte a une valeur constante aussi, mais inférieure à celle de l'eau pure, et enfin (iii) que ce changement de tension de surface intervient au moment exact du changement de vitesse.

Ce changement de tension de surface ne suffit pas à lui seul à expliquer les valeurs de vitesses obtenues dans le second régime : le rôle de l'huile (lubrification et dissipation visqueuse) est à prendre en compte également. Cependant, en s'appuyant sur la littérature, le changement de tension de surface nous permet de déduire des informations utiles sur la goutte. Une étude [6], s'intéressant à la tension de surface et l'épaisseur d'un film d'huile PDMS à la surface d'un bain d'eau, a montré que la tension de surface du bain d'eau évolue de façon surprenante en fonction du nombre de chaines libres présentes à sa surface : cette tension de surface est égale à celle de l'eau pure jusqu'à une concentration surfacique critique en chaînes de PDMS, puis chute brutalement pour atteindre un plateau au-delà de cette valeur critique. En ce qui concerne l'épaisseur du film de PDMS correspondant, en deçà de la concentration critique seuls des îlots de PDMS sont présents à la surface de l'eau, tandis qu'au-delà de la concentration critique un film recouvre toute la surface.

Le changement de régime de vitesse a lieu au moment où cette concentration critique en chaînes de PDMS est atteinte sur la goutte. Chacun des deux régimes est caractérisé par une vitesse constante : cette vitesse est différente en premier et en deuxième régime, car la surface de la goutte qui dévale est de nature différente dans chacun de ces régimes.

\section{Conséquences pratiques}

Notre étude contribue à une meilleure compréhension des interactions entre l'eau et les élastomères silicones et montre qu'une faible quantité de contaminant peut avoir des effets macroscopiques importants. Cela met en avant l'importance de s'assurer que de telles chaînes ne viennent pas perturber la conduite d'expériences où des élastomères silicones sont en contact avec de l'eau.

En outre, l'expérience que nous avons menée peut être transformée en un test simple permettant d'évaluer la présence de chaînes non réticulées dans un échantillon. Cela est possible en observant simplement la dynamique d'une goutte d'eau sur la surface que l'on souhaite tester : un choix pertinent de taille de goutte et d'angle d'inclinaison permet de visualiser les deux régimes à l'œil nu; il n'est nul besoin de disposer d'une caméra rapide.

\section{Glossaire}

Élastomère : matériau élastique formé d'un réseau de chaînes de polymères, dites réticulées car attachées entre elles par des liaisons chimiques. Ce type de matériau caoutchoutique supporte de très grandes déformations avant rupture.

Polydiméthylsiloxane (PDMS) : polymère de la famille des siloxanes, formant la structure de base de nombreuses huiles et élastomères silicones. Ce matériau, souvent présent dans les shampooings sous forme d'huile (où il est appelé diméthicone), est aussi utilisé sous forme d'élastomère (réticulé), notamment en microfluidique.

Tension de surface : force qui existe au niveau de toute interface entre deux milieux différents et qui tend à réduire l'aire de cette interface. Pour en savoir plus, consulter la référence [2].

\section{Références}

1 K. J. Regehr et al., "Biological implications of polydimethylsiloxane-based microfluidic cell culture", Lab Chip 9 (2009) 2132-2139.

2• P.-G. De Gennes, F. Brochard-Wyart et D. Quéré, Gouttes, bulles, perles et ondes (Belin, 2005).

3. A. Hourlier-Fargette et al., "Role of uncrosslinked chains in droplets dynamics on silicone elastomers", Soft Matter 13 (2017) 3484-3491.

4. J. N. Lee et al., "Solvent compatibility of poly (dimethylsiloxane)-based microfluidic devices", Anal. Chem. 75 (2003) 6544-6554.

5• K. E. Jensen et al., "Wetting and phase separation in soft adhesion", Proc. Natl. Acad. Sci. 112 (2015) 14490-14494.

6• L. T. Lee et al., "Neutron reflectivity and ellipsometry studies of a polymer molecular layer spread on the water surface", Langmuir 7 (1991) 3076-3080. 